\title{
Streamlining the investigative process - early investigation for thromboembolic and cardiovascular disease in COVID-19 patients
}

\author{
Authors: Sofia Hanger, ${ }^{\mathrm{A}}$ Josh Wall ${ }^{\mathrm{A}}$ and Linsey Christie $^{\mathrm{A}}$
}

\begin{tabular}{|c|c|c|c|c|c|}
\hline & D-dimer, $\mu g / L$ & Troponin, ng/L & BNP, ng/L & LDH, units/L & Ferritin, $\mu \mathrm{g} / \mathrm{L}$ \\
\hline Positive CTPA & $13,228(11,017-18,470)$ & $82(26-152)$ & $155(125-383)$ & $676(538-846)$ & $1,515(917-6,125)$ \\
\hline Negative CTPA & $6,325(2,428-12,034)$ & $23(15-59)$ & $164(51-443)$ & $616(51-443)$ & $1,149(706-1,887)$ \\
\hline Positive echocardiography & & $81(21-328)$ & $226(57-574)$ & & \\
\hline Negative echocardiography & & $80(22-250)$ & $152(83-363)$ & & \\
\hline
\end{tabular}

BNP = brain natriuretic peptide; CTPA = computed tomography pulmonary angiography; LDH = lactate dehydrogenase.

Early studies regarding COVID-19 have shown increased propensity for thromboembolic ${ }^{1}$ and cardiovascular ${ }^{2}$ disease. Prompt identification and treatment may reduce mortality. This project aimed to identify factors that would highlight which patients would benefit from an early computed tomography pulmonary angiography (CTPA) and echocardiography to help prioritise resources.

COVID-19-positive patients admitted to the intensive care unit (ICU) at a 430-bed general hospital from March-May 2020 were reviewed and patients with a positive CTPA or echocardiography were identified. Patient characteristics and biochemistry were analysed to help differentiate patients with positive findings.

67 COVID-19-positive patients were admitted to ICU. 32 patients underwent CTPA imaging and 32 had transthoracic echocardiographies. The positive detection rate was $47 \%$ and $50 \%$, respectively. Biochemistry results for patients with positive imaging are shown in Table 1.

$75 \%$ and $60 \%$ of patients who died did not undergo CTPA or echocardiography, respectively.

Identifying patients who benefit from CTPA and echocardiography is complex; however, D-dimer and troponin correlate with positive CTPA results (Table 1). The significant proportion of positive findings highlights the importance for high clinical suspicion for thromboembolic and cardiovascular disease.

\section{Conflicts of interest}

None declared.

\section{References}

1 Price LC, McCabe C, Garfield B, Wort SJ. Thrombosis and COVID-19 pneumonia: the clot thickens! Eur Respir J 2020;56:2001608.

2 Nishiga M, Wang DW, Han Y, Lewis DB, Wu JC. COVID-19 and cardiovascular disease: from basic mechanisms to clinical perspectives. Nat Rev Cardiol 2020;17:543-58.

Author: ${ }^{\mathrm{A} C h e l s e a ~ a n d ~ W e s t m i n s t e r ~ N H S ~ F o u n d a t i o n ~ T r u s t, ~}$ London, UK 